\title{
Experimental observation of Enhanced Plasma Potential due to unabsorbed Fast Wave on EAST
}

\author{
Xinjun Zhang ${ }^{1}$, Lunan Liu ${ }^{1,2}$, Chengming Qin ${ }^{1}$, Y. Lin ${ }^{3}$, R. Ochoukov ${ }^{4}$,Yanping Zhao ${ }^{1}$ \\ ,Noterdaeme Jean-Marie ${ }^{4,5}$, Steve wukitch ${ }^{3}$, X.Z. Gong ${ }^{1}$, B.N. Wan ${ }^{1}$, Y.T. Song ${ }^{1}$, \\ Shuai Yuan ${ }^{1}$, Hua Yang ${ }^{1}$,Yuzhou Mao ${ }^{1}$, Yan Cheng ${ }^{1}$, Lei Wang ${ }^{1}$, Xu Deng ${ }^{1}$, Gen \\ Chen $^{1}$, Songqing Ju${ }^{1}$, Kai Zhang ${ }^{1}$ and Lanlan Ping ${ }^{1,2}$ \\ ${ }^{1}$ Institute of Plasma Physics, Chinese Academy of Sciences, Hefei 230031, People's Republic \\ of China \\ ${ }^{2}$ University of Science and Technology of China, Hefei 230026, People's Republic of China \\ ${ }^{3}$ MIT Plasma Science and Fusion Center, 190 Albany St Cambridge, MA 02139, USA 4Max-Planck- \\ ${ }^{4}$ Institut für Plasmaphysik, Boltzmannstr. 2, 85748 Garching, Germany \\ ${ }^{5}$ EESA Department, University of Gent, B-9000 Gent, Belgium \\ E-mail: xjzhang@ipp.ac.cn and chmq@ipp.ac.cn
}

\begin{abstract}
An experiment on EAST is used to study the fast wave rectification by only an actively powered I-port antenna. The plasma potential up to $100 \mathrm{~V}$ was found in the region that do not map along magnetic field line to active I-port antenna. This enhancement correlates with the local fast wave intensity. An increase in the loading resistance and heating efficiency was observed at low parallel wave number. The higher heating efficiency is seen to cause lower unabsorbed fast wave power in the SOL regions. This can cause a decrease in fast wave field intensity that reaches plasma facing components and can result in decrease in the plasma potential and slow wave intensity.
\end{abstract}

Keywords: Plasma Potential, Fast Wave, Tokamak

Experimental Advanced Superconducting Tokamak (EAST) is a superconducting tokamak equipped with neutral beam injector, electron cyclotron wave, lower hybrid wave and the waves in Ion Cyclotron Range of Frequencies (ICRF)[1]. Auxiliary Heating using ICRF waves has been successful in bulk plasmas heating on EAST[2]. To maximize the ICRF heating, good coupling and strong single pass absorption are both required. The previous experimental studies of the ICRF coupling on EAST clearly show that the loading resistance and heating efficiency of the I-port antennas are strongly dependent on phasing between straps. The ICRF coupling efficiency can be improved directly by changing the antenna phasing from higher parallel wave number to lower parallel wave number [3].

The ICRF heated discharges are associated with increased levels of core ion impurity content that has been observed on limiter and divertor tokamaks, such as Tore Supra[4] JET [5] Alcator C-Mod [6] and ASDEX [7]. The typically accepted physical mechanism of this problem is believed to be ICRF sheath rectification. According to the proposed rectification theory[8-11], both the Slow wave (SW) and fast wave (FW) can lead to enhancement of the plasma potential on open magnetic field lines and can causes impurity contamination by increased impurity source, sputtering, and/or transport [12]. In both cases the plasma potential enhancement is a result of the sheath rectification. For the case of SW sheath rectification, the electric fields along the toroidal magnetic field is excited directly by the active ICRF antenna. For the case of the FW sheath rectification, the plasma potential enhancement is also due to slow wave that are generated by fast waves reflecting at conducting material surfaces. In this case, the fast wave source is in the form of unabsorbed and/or reflected fast wave from the plasma core. Most work on RF-sheath are focused on SW mechanism and simply assume the FW is subdominant. Here, we aims at FW mechanism and reveals the dominant role of the FW mechanism on EAST.

In this letter we report the first direct observation of enhanced plasma potential due to unabsorbed fast wave in the ICRF heated discharges on EAST. EAST ICRF system has a two by two strap antenna located in the B port and a four strap 
antenna in the I port[13]. All the antenna are operated in a phasing and frequency to heat a $\mathrm{H}$ minority in D plasma [2]. All the measurements were taken in the private Scrape-Off Layer(SOL) of B-port antennas using the probes located on the upper B-C side of B-port antenna limiter. Figs. 1 (a) show the top view of EAST with the ICRF antennas and the main limiter. An the view of the outer wall with the B-port antenna and relevant diagnostics used to quantify the local plasma parameters is shown in Figs.1(b). These probes are not magnetically mapped to the powered I-port antennas. The local plasma potential and the local plasma density were obtained using Langmuir probes and the local RF wave fields were measured using B dot probes[14]. The Langmuir probes are fixed behind the limiter of the B-port antenna. The location of the probes in terms of $\Delta \mathbf{R}=\boldsymbol{R}_{\text {probe }}-\boldsymbol{R}_{\text {lim }}$ is almost zero. $\boldsymbol{R}_{\text {lim }}$ is the radial location of B-port antenna limiter.
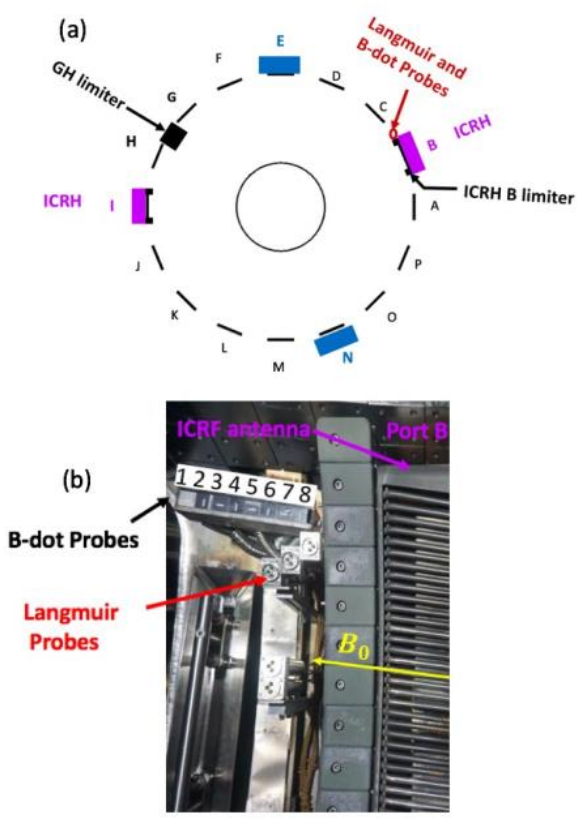

Figure1: (a) A diagram showing the top view of the EAST. ICRF antennas and The locations of key probes are shown.

(b) the view of outer wall, including B-port antenna and the relevant diagnostics.

In order to study the fast wave rectification, an experiments was performed by only an actively powered the I-port antenna in the L-mode discharge. The frequency that have been used is $34 \mathrm{MHz}$. Each toroidal strap is powered by a single transmitter and can be phased relative to the other straps. The parallel wave number of I-port atenna was varied by changing the phase between straps while keeping all other plasma parameters constant. The plasma current is $0.45 \mathrm{MA}$. The core plasma density is $3.5 \times 10^{19} \mathrm{~m}^{-3}$. The I port antenna was used with approximately $1.0 \mathrm{MW}$ power. The vacuum antenna spectrum of 78541 is peaked at $5 \mathrm{~m}^{-1}$ for the $[0 \pi / 32 \pi / 3 \pi]$ phasing. The vacuum antenna spectrum of 78545 peaked at

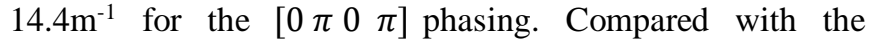
discharge 78545, as shown in Fig.2, the discharge 78541 showed a larger increase in the plasma stored energy $\left(\Delta W_{p} / \Delta P_{I C R F}=18 \mathrm{kJMW}^{-1}\right.$ versus $\left.12 \mathrm{kJMW}^{-1}\right)$ whereas , the total radiation power of 78545 is larger than 78541(Fig.2.(a) and Fig.2.(c)) . The average antenna coupling resistance increase from $2.0 \Omega$ to $2.7 \Omega$. The absorbed power increase from $0.31 \mathrm{MW}$ to $0.4 \mathrm{MW}$. Namely, ICRF heated discharge with low parallel wave number is characterized by higher coupling efficiency through evanescent layer and higher absorbed ICRF power in core plasma. It is also confirmed by neutron rate as illustrated in Fig.2(b).

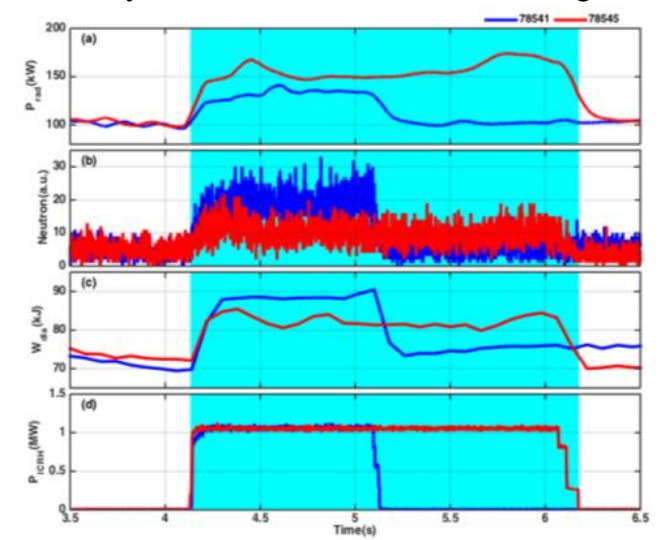

Figure2: Blue and Red lines indicate data taken in 78541 and 78545, respectively. Panel(a) illustrates the total radiation power; the neutron rate in(b); plasma stored energy in (c); in (d), we shown the injected power of I port antenna.

The correlation between the fast wave and slow wave strength for these two discharges are ploted in Fig. 3. The measurements reveal that the averaged power in the fast wave is typically a factor of 10 greater than in the slow wave. The dominant ICRF component is the fast wave component in this case. The trend for these two discharges show that a significant decrease in the strength of fast wave was obtained for higher heating efficiency. This is due to the varying fast wave absorption in the plasma core, which has an effect on unabsorbed fast wave electric field strength in the SOL regions. The higher heating efficiency can cause lower unabsorbed fast wave in the SOL regions and can result in a decrease in fast wave intensity.

An extensive experimental survey of the correlation between the plasma potential and fast wave intensity is carried out in only I-port antenna heated discharges, as shown in Fig.4. The Plasma potential up to $100 \mathrm{~V}$ are observed in the unmapped region where the active I-port antenna do not magnetically map to the probes. In all cases, the ICRFenhanced plasma potentials correlate with the strength of the fast wave. Compared to the results shown in Fig.3, both plasma potential and the local slow wave intensity correlate with the local fast wave consistent with verying the fast wave absorption in core plasma. 


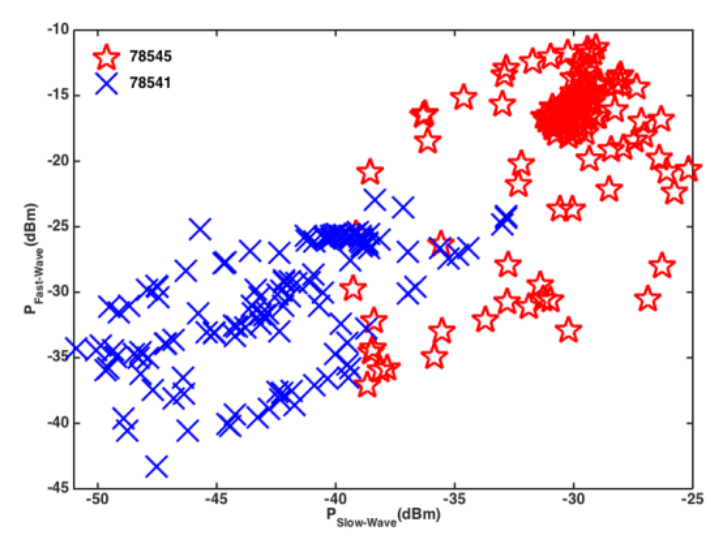

Figure 3: Correlation between the local slow wave and the local fast wave intensity for the discharges under constant plasma conditions with Prf=1.0MW. Blue and Red color indicate data taken in 78541 and 78545, respectively.

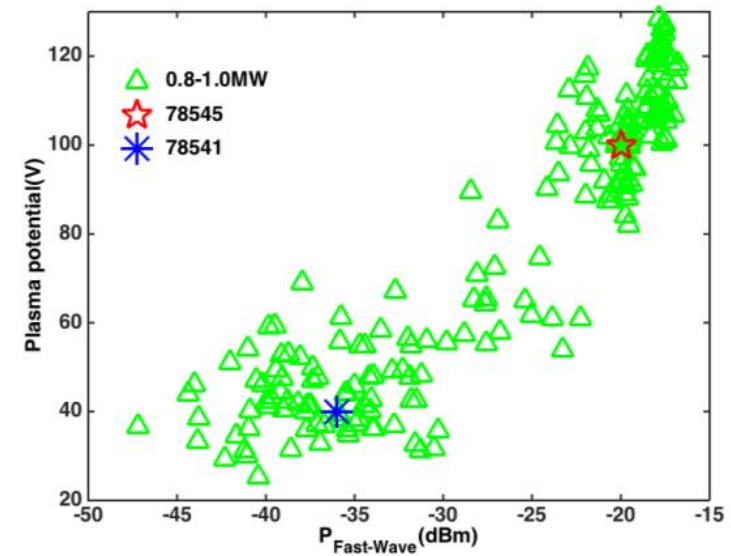

Figure 4. Correlation between ICRF enhanced plasma potential and the local Fast wave intensity for the active I-

port antenna only and RF power from $0.8-1.0 \mathrm{MW}$

The results shown in Fig. 3 and Fig.4 are consistent with the fast wave rectification mechanism[11]. The RF enhanced plasma potential in the unmapped regions would depend on the local fast wave intensity. The correlations between the plasma potential and the local fast wave intensity confirm this trend. Our observations are in agreeement with the resutls from ICRF heating experiments on Alcator C-Mod, in which fast wave absorption in the core has a dramatic effect on unabsorbed fast wave reached the SOL regions[15]. These resutes suggest that it is benefical to balance the ICRF coupling efficiency through evanescent layer and heating efficiency in the core plasma to minimize the effect of fast wave power on the material interactions.

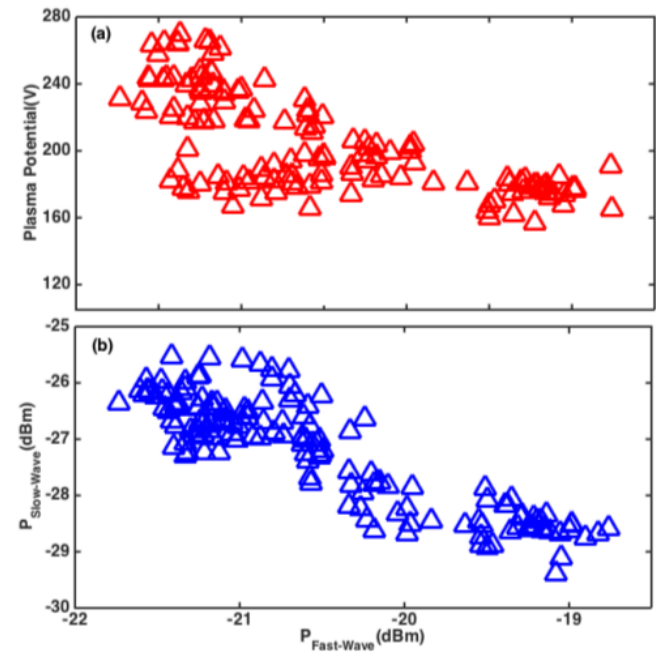

Figure 5. (a) Correlation between ICRF enhanced plasma potential and the local fast wave intensity and (b) Slow wave intensity as a function of the fast wave intensity for the active B-port antenna only

As shown in Fig.5(a), the plasma potentials in excess of $100 \mathrm{~V}$ in the SOL plasma has been observed in the B port antenna heating discharges of EAST where the active B-port antenna do magnetically map to the probes. The behavior of the plasma potential and the local fast waves intensity in Fig.5 in mapped case is in agreement with the slow wave rectification theory. The changes in the plasma potential and the slow wave intensity anti-correlates with the change in the fast wave intensity. Unlike the slow wave rectification mechanism, our experimental results in umapped case indicate that the changes in the plasma potential and slow wave intensity are correlated with the change of fast wave intensity. Compared with the results in the mapped case, fast wave rectification is a leading candidate mechanism responsible for the observed enhancement. The unabsorbed fast wave power in the core plasma can produce enhanced plamsa potential on open field line in the SOL regions.

Summary- An increase in the plasma potential up to $100 \mathrm{~V}$ are found in the region that do not map to active I-port antenna. The observed plasma potentials and slow wave intensity in unmapped locations correlate with the fast wave intensity. As antenna phaseing switch from high parallel wave number to low parallel wave number, the drop in the fast wave intensity and the plasma potential is consistent with the fast wave rectification mechanism. These experimental observations are consistent with the idea that the potential enhancements in unmaped SOL regions are the results of fast wave incident into the SOL due to unabsorbed fast wave in the core plasma

\section{Acknowledgements}

This work was supported by: 1) National key research and development program (grant No. 2016YFA0400600 and 
2016YFA0400601); 2) National Natural Science Foundation of China under grant No. 11575237, 11675213, 11375235 and 11375236; 3) National Magnetic confinement Fusion Science Programme (grant No. 2015GB101001 and 2013GB106001B).

\section{References}

[1] Zhao Y.P. et al 2014 EAST ion cyclotron resonance heating system for long pulse operation Fusion Eng. Des.

[2] Zhang X.J. et al 2013 First results from H-mode plasmas generated by ICRF heating in the EAST Nucl. Fusion 53 023004

[3] J.H. Zhang, X.J. Zhang et al Experimental analysis of the ICRF waves coupling in EAST Nucl. Fusion 57 (2017) 066030

[4] L. Colas, A. Argouarch, S. Brémond, M. Chantant, Y. Corre, M. Firdaouss, M. Goniche, D. Guilhem, J.-P. Gunn, J. Jacquot, M. Kubič, X. Litaudon, G. Lombard, O. Meyer, P. Mollard, K. Vulliez. Journal of Nuclear Materials, 438:S330—S333, 2013.

[5] Vl. Bobkov, G. Arnoux, S. Brezinsek, J.W. Coenen, L. Colas, M. Clever, A. Czarnecka, F. Braun, R. Dux, A. Huber, P. Jacquet, C. Klepper, E. Lerche, C. Maggi, F. Marcotte, M. Maslov, G. Matthews, M.L. Mayoral, K. McCormick, A. Meigs, D. Milanesio, I. Monakhov, R. Neu, J.- M. Noterdaeme, Th. Pütterich, F. Rimini, G. Van Rooj, G. Sergienko, D. Van Eester, JET EFDA contributors. Journal of Nuclear Materials, 438:S160-S165, 2013.

[6] R. Neu, A. Kallenbach, M. Balden, V. Bobkov, J.W. Coenen, R. Drube, R. Dux, H. Greuner, A. Herrmann, J. Hobirk, H. Höhnle, K. Krieger, M. Kočan, P. Lang, T. Lunt, H. Maier, M. Mayer, H.W. Müller, S. Potzel, T. Pütterich, J. Rapp, V. Rohde, F. Ryter, P.A. Schneider, J. Schweinzer, M. Sertoli, J. Stober, W. Suttrop, K. Sugiyama, G. van Rooij, M. Wischmeier, ASDEX Upgrade Team. Journal of Nuclear Materials, 438:S34-S41, 2013.

[7] V. Bobkov, F. Braun, R. Dux, A. Herrmann, L. Giannone, A. Kallenbach, H.W. Muller, R. Neu, J.-M. Noterdaeme, T. Putterich, V. Rohde, A. Sips, A. Krivska, I. Zammuto and ASDEX Upgrade Team. 24th IAEA Fusion Energy Conference Proceedings, 2012.

[8] J.R. Myra, D.A. D'Ippolito, and M. Bures. Physics of Plasmas; 1:2890-2900, 1994.

[9] D.A. D’Ippolito, J.R. Myra, E.F. Jaeger, and L.A. Berry. Physics of Plasmas; 15:102501- 12, 2008.

[10] D.A. D'Ippolito, J.R. Myra, R. Ochoukov and D.G. Whyte. Plasma Physics and Controlled Fusion; 55:085001, 2013.

[11] J.R. Myra, D.A. D’Ippolito, Phys. Rev. Lett. 101 (2008) 195004.

[12] B.Lipschultz, D.A.Pappas et al A study of molybdenum influx and transport in Alcator C-Mod Nucl. Fusion 41 585-596

[13] Qin C., Zhang X., Zhao Y., Wan B., Braun F., Wang L., Yang Q., Yuan S., Cheng Y. and ICRF team Electromagnetic analysis of the EAST 4-strap ICRF antenna with HFSS code Plasma Sci. Technol. 17 167-72

[14] Liu Lunan Xinjun Zhang et al, High-frequency B-dot probes used to detect characteristics of ion cyclotron range-of frequency waves in EAST, Journal of Plasma Physics, Accepted
[15] R. Ochoukov, D.G. Whyte, D.Brunner, I. Cziegler et al Investigation of RF enhanced plasma potentials on Alcator CMod Journal of Nuclear Materials , 2013, 438 :S875-S878 